\title{
Mild Hypothermia and Neurologic Outcomes in Patients undergoing Venoarterial Extracorporeal Membrane Oxygenation
}

\author{
Sung-Min Cho ${ }^{1}$, Mais Al-Kawaz ${ }^{1}$, Benjamin Shou ${ }^{2}$, Rochelle Prokupets ${ }^{1}$, Glenn Whitman ${ }^{2}$, \\ and Romergryko Geocadin ${ }^{1}$ \\ ${ }^{1}$ Johns Hopkins Medicine Department of Anesthesiology and Critical Care Medicine \\ ${ }^{2}$ Johns Hopkins Medicine Department of Surgery
}

November 26, 2021

\begin{abstract}
Background: Patients with venoarterial extracorporeal membrane oxygenation (VA-ECMO) are at risk of cerebral reperfusion injury after prolonged hypoperfusion and immediate restoration of systemic blood flow. We aimed to examine the impact of mild hypothermia during the first 24 hours post-ECMO on neurological outcome in VA-ECMO patients. Methods: This was a retrospective study of adult VA-ECMO patients from a tertiary care center. Mild hypothermia was defined as $32-36^{\circ} \mathrm{C}$ during the first 24 hours post-ECMO. Primary outcome was good neurological function at discharge measured by a modified Rankin Scale [?]3. Multivariable logistic regression analysis was performed for primary outcome adjusting for pre-specified covariates. Results: Overall, 128 consecutive patients with VA-ECMO support (median age: 60 years and 63\% males) were included. Within the first 24 hours of VA-ECMO cannulation, we found a median of 71 readings per patient (interquartile range 45-88). Eighty-eight patients (68.8\%) experienced mild hypothermia within the first 24 hours while 18 of those 88 patients (14.2\%) had a mean temperature<36degC. ECMO indications included post-cardiotomy shock (39.8\%), cardiac arrest (29.7\%), and cardiogenic shock $(26.6 \%)$. Duration of mild hypothermia, but not mean temperature, was independently associated with increased odds of good neurological outcome at discharge (Odds Ratio $[\mathrm{OR}]=1.16,95 \%$ Confidence Interval $[\mathrm{CI}]=1.04-1.31, \mathrm{p}=0.01$ ) after adjusting for age, severity of illness, post-ECMO systemic hemorrhage, post-cardiotomy shock, acute brain injury, and mean 24-hour $\mathrm{PaO}_{2}$. Neither duration of mild hypothermia $(\mathrm{OR}=0.93, \mathrm{CI}=0.84-1.03, \mathrm{p}=0.17)$ nor mean temperature $(\mathrm{OR}=0.78$, $\mathrm{CI}=0.29-2.08, \mathrm{p}=0.62)$ was significantly associated with mortality. Similarly, duration of mild hypothermia $(\mathrm{p}=0.47)$ and mean 24-hour temperature $(\mathrm{p}=0.76)$ were not significantly associated with frequency of systemic hemorrhages. Conclusions: In this single center study, longer duration of mild hypothermia during the first 24 hours of ECMO support was significantly associated with improved neurological outcome. Mild hypothermia was not associated with an increased risk of systemic hemorrhage or improved survival.
\end{abstract}

\section{Introduction}

Therapeutic hypothermia has been shown to improve neurological outcome and mortality in comatose cardiac arrest patients. ${ }^{1-3}$ Patients undergoing extracorporeal membrane oxygenation (ECMO) constitute a special population with a high risk of acute brain injury (ABI), up to $15.1 \%$ in patients undergoing veno-arterial (VA) ECMO, and $7.1 \%$ in patients undergoing veno-venous ECMO. ${ }^{4}$ While evidence for improving neurological outcome with therapeutic hypothermia $32-36^{\circ} \mathrm{C}$ is well delineated in cardiac arrest,, $15-9$ the effect of hypothermia in VA-ECMO is unknown. VA-ECMO patients are susceptible to reperfusion injury given their prolonged hypoperfusion preceding full restoration of cerebral blood flow, which may benefit from hypothermia. However, hypothermia may increase the risk of hemorrhagic events with acquired coagulopathy in ECMO. ${ }^{10}$ We hypothesized that mild hypothermia during the first 24 hours of ECMO may improve neurological outcome without exacerbating the frequency of systemic hemorrhages.

\section{Methods}


Population: We performed a retrospective analysis of ECMO patients admitted to the Johns Hopkins Hospital between 2016 and 2019. We included all adult patients (age $>18$ years), excluding patients who underwent multiple runs to minimize the potential bias resulting from severe illness. This study was carried out with the approval of the Johns Hopkins institutional review board (IRB00216321). Informed consent and clinical trial registration are not applicable for this study. Demographics, past medical history, laboratory values, ECMO indication, hemodynamic data, and Sequential Organ Failure Assessment (SOFA) score (day 1) were collected. ${ }^{11} \mathrm{ABI}$ included ischemic stroke, intracranial hemorrhage (ICH), cerebral edema, hypoxic ischemic brain injury, seizures, and coma (Glasgow Coma Scale $<8$ ) despite being off sedation for $>24$ hours. ${ }^{12}$ Systemic hemorrhage included surgical site bleeding, gastrointestinal bleeding, disseminated intravascular coagulation, pulmonary hemorrhage, and genitourinary bleeding.

Exposure: Temperature measurements were collected during the first 24 hours after ECMO cannulation. Bladder temperatures were collected hourly, while additional recordings were obtained when clinically indicated. Metrics for hypothermia included: mean temperature and time (hours) spent between $32-36^{\circ} \mathrm{C} .{ }^{5}$

Outcomes: The primary outcome was neurological function at discharge defined by modified Rankin Scale $(\mathrm{mRS})$, with a score of 0-3 defined as good neurological outcome (4-6: poor). ${ }^{13}$ Secondary outcomes included in-hospital mortality and systemic hemorrhage.

Statistical analysis: Demographic and clinical variables for hypothermia vs. no hypothermia were compared by unpaired t-test, chi-squared test, or Mann-Whitney U test as appropriate. Results were expressed as the mean with standard deviation if normally distributed or as the median with quartiles if non-normally distributed for quantitative variables and as proportions for categorical variables. Carefully selected prespecified variables a priori that showed significant association with primary outcome in univariate analyses were used in the multivariable models reported. A p value $<0.05$ was considered statistically significant. Odds ratios (ORs) with 95\% confidence intervals (CIs) were reported. All analyses were carried out in STATA 15 (College Station, TX, USA).

\section{Results}

Of the 128 patients with VA-ECMO support, the median age was 60 years (interquartile range [IQR]: 49.5$68.5)$ and 81 (63\%) were males. ECMO indications included post-cardiotomy shock (39.8\%), cardiac arrest (29.7\%), and cardiogenic shock (26.6\%). Of 128, 66 (51.6\%) experienced ABI.

Within first 24 hours, we found 8,423 temperature measurements(Supplemental Figure 1) with a median of 71 readings per patient. Mild hypothermia of varying durations was observed in 88/128 patients (68.8\%) within the first 24 hours of ECMO support, and 18/128 patients (14.2\%) had a mean temperature less than $36^{\circ} \mathrm{C}$ (range: $32.4-35.9^{\circ} \mathrm{C}$ ). Mean 24-hour temperature was $36.4^{\circ} \mathrm{C}$ within the post-cardiotomy shock patients, $36.0^{\circ} \mathrm{C}$ within the cardiac arrest patients, and $36.5^{\circ} \mathrm{C}$ within the cardiogenic shock patients.

Baseline characteristics of patients with vs. without mild hypothermia were comparable (Table 1 ). 5/18 $(27.8 \%)$ patients who had good neurologic outcome with mild hypothermia, while 21/110 patients who had good neurologic outcome without mild hypothermia (19.1\%) ( $\mathrm{p}=0.40)$ (Supplemental Figure 2 ).

In a multivariable model adjusting for age, SOFA score, ABI, systemic hemorrhage, mean 24-hour $\mathrm{PaO}_{2}$, and post-cardiotomy shock, mean 24-hour temperature was not associated with good neurological outcome $(\mathrm{OR}=0.70,95 \% \mathrm{CI}=0.18-2.73, \mathrm{p}=0.61)$. Using the same model, longer duration of mild hypothermia was independently associated with good neurological outcome $(\mathrm{OR}=1.17,95 \% \mathrm{CI}=1.04-1.31, \mathrm{p}=0.01)$ (Figure 1, Table 2 ).

In a sensitivity analysis, neither duration of mild hypothermia $(\mathrm{OR}=0.93,95 \% \mathrm{CI}=0.84-1.03, \mathrm{p}=0.17)$ nor mean temperature $(\mathrm{OR}=0.78,95 \% \mathrm{CI}=0.29-2.08, \mathrm{p}=0.62)$ was significantly associated with mortality. Similarly, duration of mild hypothermia $(\mathrm{p}=0.47)$ and mean 24 -hour temperature $(\mathrm{p}=0.76)$ were not significantly associated with frequency of systemic hemorrhages (Supplemental Results, Supplemental Table 1-4) - After excluding patients who experienced cardiac arrest prior to VA-ECMO $(\mathrm{n}=58)$, longer duration of mild hypothermia was associated with improved neurologic outcome at discharge, however this finding was 
not statistically significant $(\mathrm{OR}=0.81,95 \% \mathrm{CI}=0.64-1.01, \mathrm{p}=0.07)$. Mortality was not associated with either mean 24-hour temperature $(\mathrm{OR}=0.77, \mathrm{CI}=0.28-2.09, \mathrm{p}=0.62)$ (Supplemental Table 1) or duration of mild hypothermia $(\mathrm{OR}=0.93, \mathrm{CI}=0.85-1.03, \mathrm{p}=0.17)$ (Supplemental Table 2) after adjusting for age, SOFA score, ABI, mean 24-hour $\mathrm{PaO}_{2}$, and post-cardiotomy shock.

Overall, 68 (54\%) experienced systemic hemorrhages. Patients with systemic hemorrhage were more likely to have higher SOFA scores $(\mathrm{p}=0.003)$, more likely to be centrally cannulated $(\mathrm{p}=0.02)$, and more likely to have post-cardiotomy shock $(\mathrm{p}=0.04)$. In a multivariable model adjusting for SOFA scores, central cannulation, and post-cardiotomy shock, mean 24-hour temperature $(\mathrm{OR}=0.91, \mathrm{CI}=0.53-1.57, \mathrm{p}=0.73)$ (Supplemental Table 3) and duration of hypothermia $(\mathrm{OR}=0.97, \mathrm{CI}=0.90-1.05, \mathrm{p}=0.48)$ (Supplemental Table 4) was not associated with increased odds of systemic hemorrhage. Eight $(6.3 \%)$ had ICH and mean temperature $(\mathrm{p}=0.30)$ and duration of hypothermia was not associated with ICH $(\mathrm{p}=0.99)$.

\section{Discussion}

In this paper, we found a significant association between longer duration of mild hypothermia and good neurological outcome at discharge. This finding supports the hypothesis that early mild hypothermia exerts a positive effect on neurological outcome in VA-ECMO patients. This observation stands true even after excluding post-cardiotomy patients who did not experience cardiac arrest. Interestingly, mild hypothermia did not influence in-hospital mortality. Although one may hypothesize that bleeding may be exacerbated with ECMO-associated coagulopathy in addition to hypothermia, this was not found to be the case.

We previously showed that early hyperoxia was a strong marker of poor neurological outcome in VA-ECMO patients. ${ }^{14}$ In this study, hypothermia proved to be yet another significant factor in neurological outcome, after adjusting for important risk factors including hyperoxia, suggesting temperature management may be an early intervention that improves neurological outcome in this population. Nevertheless, despite our findings, there is conflicting evidence regarding the benefit of therapeutic hypothermia in ECMO patients. In agreement with our study, in a pooled analysis of 13 studies, Chen et al. found a significant association between hypothermia $\left(32-34^{\circ} \mathrm{C}\right)$ and favorable neurologic outcomes, defined by a cerebral performance category of $1-2 .{ }^{15}$ In contrast, a recent meta-analysis of 35 studies showed that among extracorporeal cardiopulmonary resuscitation (eCPR) patients, survival and neurological outcomes were not different between patients who underwent therapeutic hypothermia (ranging between $33-36^{\circ} \mathrm{C}$ ) and patients who did not. ${ }^{16}$ However, these reports, unlike our study, were limited by high heterogeneity of included studies without granular temperature data.

Furthermore, the data on the effect of mild hypothermia in non-eCPR VA-ECMO patients is sparse. While limited data exists regarding the benefit of early, therapeutic hypothermia in ECMO patients, a physiologic explanation for why it might be beneficial can be opined for all 3 cohorts, eCPR, cardiogenic shock, and post-cardiotomy shock. Reperfusion injury secondary to prolonged low flow in eCPR patients portends a significant neurological injury, which may be mitigated by TTM. ${ }^{17}$ Also, hypothermia may offer hemodynamic benefits following cardiogenic shock including reduced metabolic rate, increased contractility, and increased cardiac output. ${ }^{18,19}$ A similar advantageous hemodynamic profile could result in improved neurological outcomes in post-cardiotomy shock patients. This study provides supporting evidence that even in the non-eCPR patients, mild hypothermia was associated with good neurological outcome. Therefore, our study is hypothesis-generating, necessitating further research on hypothermia in each VA-ECMO indication.

This study has several limitations. It is a single center observational study. We included ECMO patients with different indications such as cardiac arrest, cardiogenic shock, and post-cardiotomy shock. However, we performed a sensitivity analysis to exclude patients with cardiac arrest and the and the benefit to hypothermia persisted. Given the limited sample size, a multi-center study is necessary to study this question in each VAECMO indication. Despite the small sample size, a beneficial effect of hypothermia was large on neurological outcome.

\section{Conclusions}


In patients undergoing VA-ECMO, longer duration of hypothermia during the first 24 hours of ECMO support was significantly associated with improved neurological outcome at discharge. Further prospective multicenter research is warranted to validate these findings.

\section{Figure Legends}

Figure 1. Linear model of probability of good neurologic outcome at discharge as a function of duration of hypothermia (a) and mean temperature (b) with $95 \%$ confidence intervals.

\section{References}

1. Hypothermia after Cardiac Arrest Study G. Mild therapeutic hypothermia to improve the neurologic outcome after cardiac arrest. $N$ Engl $J$ Med. 2002;346(8):549-556.

2. Nielsen N, Wetterslev J, Cronberg T, et al. Targeted temperature management at 33 degrees $\mathrm{C}$ versus 36 degrees C after cardiac arrest. N Engl J Med. 2013;369(23):2197-2206.

3. Nichol G, Huszti E, Kim F, et al. Does induction of hypothermia improve outcomes after in-hospital cardiac arrest? Resuscitation.2013;84(5):620-625.

4. Cho SM, Canner J, Chiarini G, et al. Modifiable Risk Factors and Mortality From Ischemic and Hemorrhagic Strokes in Patients Receiving Venoarterial Extracorporeal Membrane Oxygenation: Results From the Extracorporeal Life Support Organization Registry. Crit Care Med.2020;48(10):e897-e905.

5. Donnino MW, Andersen LW, Berg KM, et al. Temperature Management After Cardiac Arrest: An Advisory Statement by the Advanced Life Support Task Force of the International Liaison Committee on Resuscitation and the American Heart Association Emergency Cardiovascular Care Committee and the Council on Cardiopulmonary, Critical Care, Perioperative and Resuscitation. Resuscitation. 2016;98:97-104.

6. Bernard SA, Gray TW, Buist MD, et al. Treatment of comatose survivors of out-of-hospital cardiac arrest with induced hypothermia. N Engl J Med. 2002;346(8):557-563.

7. Testori C, Sterz F, Behringer W, et al. Mild therapeutic hypothermia is associated with favourable outcome in patients after cardiac arrest with non-shockable rhythms. Resuscitation. 2011;82(9):1162-1167.

8. Perman SM, Grossestreuer AV, Wiebe DJ, Carr BG, Abella BS, Gaieski DF. The Utility of Therapeutic Hypothermia for Post-Cardiac Arrest Syndrome Patients With an Initial Nonshockable Rhythm.Circulation. 2015;132(22):2146-2151.

9. Lascarrou JB, Merdji H, Le Gouge A, et al. Targeted Temperature Management for Cardiac Arrest with Nonshockable Rhythm. N Engl J Med. 2019;381(24):2327-2337.

10. Aubron C, DePuydt J, Belon F, et al. Predictive factors of bleeding events in adults undergoing extracorporeal membrane oxygenation. Ann Intensive Care. 2016;6(1):97.

11. Vincent JL, de Mendonca A, Cantraine F, et al. Use of the SOFA score to assess the incidence of organ dysfunction/failure in intensive care units: results of a multicenter, prospective study. Working group on "sepsisrelated problems" of the European Society of Intensive Care Medicine. Crit Care Med. 1998;26(11):1793-1800.

12. Hunt MF, Clark KT, Whitman G, Choi CW, Geocadin RG, Cho SM. The Use of Cerebral NIRS Monitoring to Identify Acute Brain Injury in Patients With VA-ECMO. J Intensive Care Med. 2020:885066620966962.

13. Bonita R, Beaglehole R. Recovery of motor function after stroke.Stroke. 1988;19(12):1497-1500.

14. Al-Kawaz MN, Canner J, Caturegli G, et al. Duration of Hyperoxia and Neurologic Outcomes in Patients Undergoing Extracorporeal Membrane Oxygenation. Crit Care Med. 2021.

15. Chen X, Zhen Z, Na J, Wang Q, Gao L, Yuan Y. Associations of therapeutic hypothermia with clinical outcomes in patients receiving ECPR after cardiac arrest: systematic review with meta-analysis.Scand $J$ 
Trauma Resusc Emerg Med. 2020;28(1):3.

16. Huang M, Shoskes A, Ibrahim M, et al. Does Targeted Temperature Management Improve Neurological Outcome in Extracorporeal Cardiopulmonary Resuscitation (ECPR)? J Intensive Care Med.2021:8850666211018982.

17. Wei H, Yin M, Lu Y, et al. Mild hypothermia improves neurological outcome in mice after cardiopulmonary resuscitation through Silent Information Regulator 1-actviated autophagy. Cell Death Discov.2019;5:129.

18. Nishimura Y, Naito Y, Nishioka T, Okamura Y. The effects of cardiac cooling under surface-induced hypothermia on the cardiac function in the in situ heart. Interact Cardiovasc Thorac Surg.2005;4(2):101105.

19. Weisser J, Martin J, Bisping E, et al. Influence of mild hypothermia on myocardial contractility and circulatory function. Basic Res Cardiol. 2001;96(2):198-205.

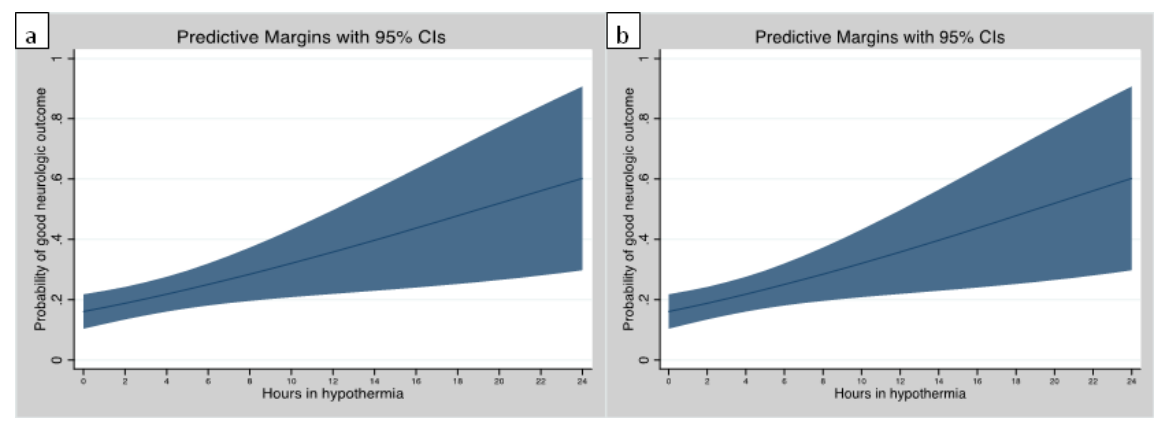

\section{Hosted file}

Tables.docx available at https://authorea.com/users/448148/articles/547005-mild-hypothermiaand-neurologic-outcomes-in-patients-undergoing-venoarterial-extracorporeal-membraneoxygenation 